\title{
Recovering the ages and metallicities of stars of a complex stellar population system
}

\author{
Sebastian L. Hidalgo ${ }^{1}$, Antonio Aparicio ${ }^{1,2}$, and Carme Gallart ${ }^{1}$ \\ ${ }^{1}$ Instituto de Astrofísica de Canarias, Vía Láctea, La Laguna, Tenerife, Canary Islands, Spain \\ ${ }^{2}$ Departamento de Astrofísica, Universidad de La Laguna, Tenerife, Canary Island, Spain
}

\begin{abstract}
We present a new method to solve for the star-formation history (SFH) of a complex stellar population system from the analysis of the color-magnitude diagram (CMD). The SFH is obtained in four steps: i) computing a synthetic CMD, ii) simulating observational effects, iii) parameterization and sampling of the synthetic and observed CMDs, and iv) solving and averaging the solutions. The consistency and stability of the method have been tested using a mock stellar population.

The method has been used to solve the SFH of a set of six isolated Local Group dwarf galaxies observed with HST. The main goal is to probe the effects of cosmological processes, such as reionization in the early star formation, or the ability of SNe feedback to remove gas in small halos, in dwarf galaxies free from environmental effects due to the strong interaction with the host galaxy.
\end{abstract}

Keywords. Hertzsprung-Russell diagram, galaxies: stellar content, (galaxies:) Local Group, methods: numerical

\section{Introduction}

The color-magnitude diagram (CMD) is the best tool to derive the star formation history $(\mathrm{SFH})$ of resolved galaxies. If the $\mathrm{CMD}$ is deep enough, stars born all over the life-time of the galaxy can be observed. However, deciphering an accurate SFH in a complex stellar population system requires some relatively sophisticated techniques. The most extended, and probably most powerful, technique is the one based on synthetic CMD analysis. We present a procedure and a set of algorithms designed for this task. The main code we present here is based in the same principle as that used by Aparicio, Gallart, \& Bertelli (1997) for the solution convergence. Applied in the most general way, it derives the SFR of a system as a function of both time and metallicity, from the comparison of its CMD star distribution with the star distribution in a synthetic CMD.

\section{Basic concepts and definitions}

We will adopt here the following approach: considering that time and metallicity are the most important variables in the problem, we define the SFH as a function $\psi(t, z)$ such that $\psi(t, z) \mathrm{d} t \mathrm{~d} z$ is the number of stars formed at time $t^{\prime}$ in the interval $t<t^{\prime} \leqslant t+\mathrm{d} t$ and with metallicity $z^{\prime}$ in the interval $z<z^{\prime} \leqslant z+\mathrm{d} z$, per unit time and metallicity. $\psi(t, z)$ is a distribution function and can be identified with the usual SFR, but as a function both of time and metallicity.

There are several other functions and parameters related to the $\mathrm{SFH}$, that we will consider here as auxiliary. The initial mass function (IMF), $\phi(m)$, and a function accounting for the frequency and relative mass distribution of binary stars, $\beta(f, q)$, are the main 
ones. The solution found for the SFH depends on the assumptions made for these functions. The method we present here uses several choices of both with the aim of setting constraints on them.

Other parameters affecting the solution of $\psi(t, z)$ are distance and reddening, including differential reddening. But the strongest limitation on the observational information is produced by the observational effects. These include all the factors affecting and distorting the observational material, namely signal-to-noise limitations, defects on the detector, and crowding and blending of stars. The consequences are loss of stars, changes in measured stellar colors and magnitudes, and external errors larger and more difficult to control than internal ones.

\section{Running the algorithms}

The method is designed to obtain a SFH in four steps, each one, handled by an algorithm:

- Generating one (or several) global synthetic stellar population, which serves as a model (algorithm: IAC-star).

- Simulating observational effects on the model (obsersin).

- Parameterization and sampling the model and data (minniac).

- Solving the equations (IAC-pop).

\subsection{IAC-star}

IAC-star (Aparicio \& Gallart 2004) is a code for synthetic CMD computation. In short, the algorithm is intended to be as general as possible and allows a variety of inputs for the initial mass function (IMF), star formation rate, metallicity law, and binarity. IAC-star is used to generate a global synthetic stellar population with a large number of stars with ages and metallicities with a constant distribution over the full interval of variation of $\psi(t, z)$ in time and metallicity. If functions $\phi(m), \beta(f, q)$ are to be explored, a global synthetic stellar population must be generated for each choice of $\phi(m)$ and $\beta(f, q)$, as mentioned before. Among other quantities, IAC-star provides magnitudes in several filters, age, and metallicity for each synthetic star, and the integral of the SFH (i.e. total mass ever formed in the model), used to normalize the solutions. Synthetic magnitudes are used to plot the CMD (sCMD) associated with the global synthetic population.

\subsection{Obsersin}

Once the sCMD is generated, observational effects must be simulated. This is done by obsersin which makes use of the results of previously done completeness tests. The completeness tests are done by injecting a list of false stars in the observed images and recovering them using the same photometric procedure used for the photometry of real stars. From the list of the unrecovered stars and the differences between the injected $\left(m_{i}\right)$ and recovered magnitudes $\left(m_{r}\right)$ obsersin uses the following procedure to simulate the observational effects: for any star from sCMD (called a 'synthetic star') with magnitude $m_{s}$, a list of false stars with $\left|m_{i}-m_{s}\right| \leqslant \epsilon$ is created for each filter, with $\epsilon$ being a free input searching interval. From the stars in common to both filters, a single false star is selected by a simple random sampling. If $m_{i}^{\prime}$ and $m_{r}^{\prime}$ are the injected and recovered magnitudes of the selected false star in a given filter, then $m_{s}^{e}=m_{s}+m_{i}^{\prime}-m_{r}^{\prime}$ will be the magnitude of the synthetic star with observational effects simulated. The same is done for both filters.

With the procedure described above, the observational effects are simulated in $\mathrm{SCMD}$ star by star. The number of injected stars in the completeness tests, in comparison with 
the number of synthetic stars in sCMD, determines the quality of the simulation of the observational effects.

\subsection{Minniac}

IAC-pop uses a method introduced by Aparicio et al. (1997) to solve the SFH. The method assumes that any SFH can be given as a linear combination of simple populations (see §3.4). We define a simple stellar population by a number of stars with ages and metallicities within small intervals. Each simple population has a CMD associated that we call the partial model CMD (pCMD). With this definition, sCMD is formed for the sum of all pCMD. To compare pCMD with the observed CMD (oCMD) we create a set of boxes and count stars in them. This process is repeated for all the pCMD included in sCMD and gives the arrays $M_{i}^{j}$, containing the number of stars from partial model $i$ populating the pCMD box $j$, and $O^{j}$, containing the number of observed stars in box $j$.

To minimize the dependency of the results on the selection of simple populations and on the size and position of the boxes, the process is repeated by introducing offsets in age and metallicity bins which define the simple populations. The age and metallicity interval for each simple population is fixed. For each new set of simple populations, the boxes can change in size and position.

In addition, for each set of simple populations and/or size and position of the sampling boxes, minniac can introduce an offset in color and magnitude in oCMD. For each new color-magnitude point, the process described above is repeated. This produces a manifold of input files which are used by IAC-pop (see below) to solve the SFH. We call model parameterization to each set of simple populations, position and size of boxes, and colormagnitude point. Each model parameterization has associated with it the arrays defined above, $M_{i}^{j}$ and $O^{j}$.

\subsection{IAC-pop}

With the information provided by minniac, the distribution of stars in the CMDs can be calculated for any model SFH as a linear combination of the $M_{i}^{j}$ calculated above:

$$
M^{j}=A \sum_{i} \alpha_{i} M_{i}^{j}
$$

It should be noted that $\alpha_{i} \geqslant 0 . A$ is a scaling constant.

The SFH best matching the distribution, $O^{j}$, of the observational CMD can be found using a merit function. In particular Mighell's $\chi_{\gamma}^{2}$ (Mighell 1999) is used:

$$
\chi_{\gamma}^{2}=\sum_{j} \frac{\left(O^{j}+\min \left(O^{j}, 1\right)-M^{j}\right)^{2}}{O^{j}+1}
$$

We will use $\chi_{\nu}^{2}=\chi_{\gamma}^{2} / \nu$, where $\nu$ is the number of degrees of freedom. In our case $\nu=k-1$, where $k$ is the number of boxes defined in the CMD. Minimization of $\chi_{\nu}^{2}$ provides the best solution as a set of $\alpha_{i}$ values. IAC-pop makes use of a genetic algorithm (Charbonneau 1995) for efficient searching of the $\chi_{\nu}^{2}$ minimum.

The solution SFH can be written as:

$$
\psi(t, z)=A \sum_{i} \alpha_{i} \psi_{i}
$$

where $\psi_{i}$ refers to partial model $i$, with $i$ taking values from 1 to $n \times m$, and $A$ is again a scaling constant. 
All solutions given by IAC-pop for the same color-magnitude point are averaged. The solutions obtained for different color-magnitude points are used to calculate external uncertainties. To find the color-magnitude offset which gives the best $\chi_{\nu}^{2}$, several choices of color-magnitude points must be explored. This procedure minimizes the impact of the uncertainties from the reddening and distance determination and the differences between the evolutionary models and data.

\section{Consistency test}

To test the consistency and stability of the method, a mock stellar population has been computed using IAC-star and analyzed with the procedure described in $\S 3$ to obtain its SFH as if it were a real one. Results have been compared with the input used to compute the mock population. The IAC-star input parameters used to compute the mock population were as follows. The BaSTI stellar evolution library (Pietrinferni et al. 2004) and the Castelli \& Kurucz (2003) bolometric correction library were used. The number of stars in the associated mock CMD (mCMD) was $10^{5}$. The star formation ranges from 14 Gyr ago to the present, with a constant SFR, $\psi(t)$, for that period. The metallicity increases with time, with initial and final metallicities $z_{0}=0.0001$ and $z_{f}=0.008$ and some metallicity dispersion at each time. Finally, no binary stars were considered and the IMF by Kroupa, Tout, \& Gilmore (1993) was used. The integral of $\psi(t, z)$ (i.e. the total mass ever transformed into stars) for this system is $\Psi_{T}=2.02 \times 10^{6} M_{\odot}$. The $\mathrm{SFH} \psi(t, z)$ of the mock population is shown in Fig. 1 on the left panel. The volume below the curved surface and over the age-metallicity plane gives the mass that has been ever transformed into stars within the considered age-metallicity interval. The SFR as a function of time only, $\psi(t)$, and of metallicity only, $\psi(z)$, are also shown. The associated $\mathrm{mCMD}$ is shown in the right panel.

Figure 2 shows on the left panel the $\psi(t, z)$ solution for the mock population given in Fig. 1. Agreement is good, being the differences between input and solution within the error bars, showing the algorithm's robustness against observational effects. The right panel shows the CMD corresponding to the solution.

Table 1 summarizes the consistency test. Column 1 identifies the CMD. Column 2 gives the $\chi_{\nu}^{2}$ value of the solution. Columns 3,4 and 5 give, respectively, the total mass $\left(M_{T}\right)$, mean age $(<$ age $\rangle)$, and mean metallicity $(\langle z\rangle)$ of the stars in the mock population (first row) and the solutions. The agreement of the integral and average values between the mock population and the solution is good in all cases.

Table 1. Results for the self-consistency test

\begin{tabular}{lcccc}
\hline \hline CMD & $\chi_{\nu}^{2}$ & $M_{T}\left(10^{6} M_{\odot}\right)$ & $<$ age $>(\mathrm{Gyr})$ & $\langle z\rangle$ \\
\hline Mock & & 2.02 & 7.00 & 0.0026 \\
Solution & 0.9 & $2.00 \pm 0.02$ & $6.91 \pm 1.10$ & $0.0026 \pm 0.0005$ \\
\hline
\end{tabular}

\section{SFHs of the LCID project}

The procedure described in the previous sections have been applied to LCID (Local Cosmology from Isolated Dwarfs) project. LCID was granted 110 orbits on the HST@ACS to obtain deep photometry of five Local Group isolated dwarf galaxies, each with a different level of current star formation activity and gas fraction: IC1613, Leo A, Tucana, LGS 3 and Cetus. The Phoenix dwarf galaxy was observed previously with the HST@WFPC2 

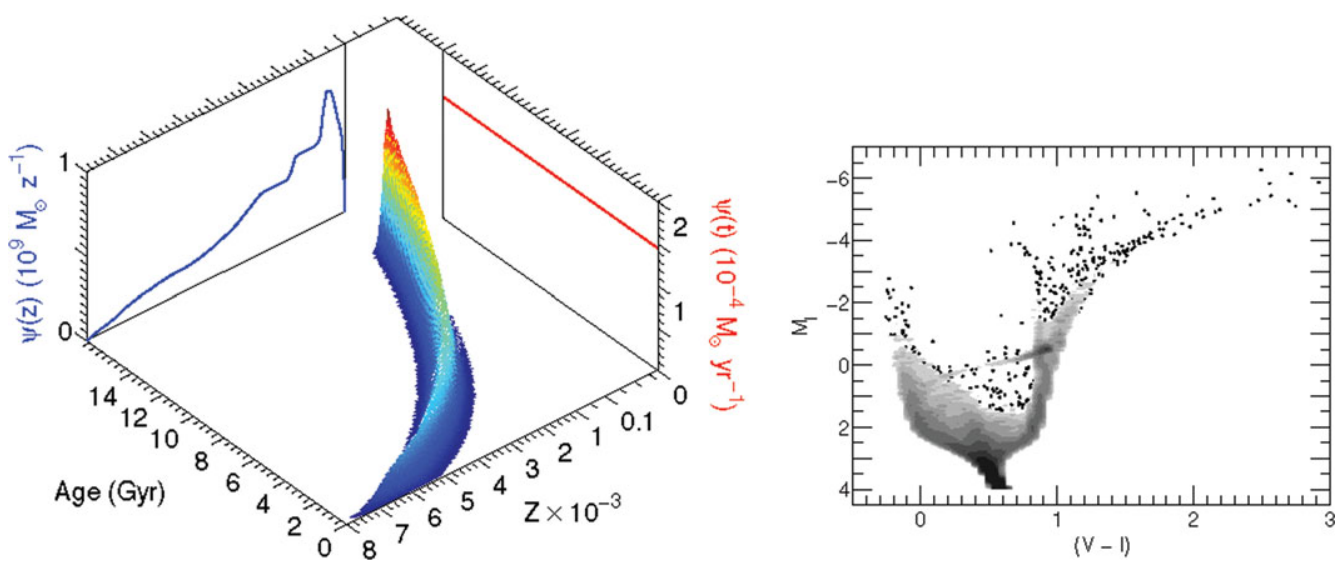

Figure 1. The SFH $\psi(t, z)$ of the mock population (left panel). Age and metallicity are given in the horizontal axis. The volume below the curved surface and over the age-metallicity plane gives the mass that has been ever transformed into stars within the considered age-metallicity interval. The mono-dimensional $\psi(t)$ and $\psi(z)$ are shown on the $\psi$-age plane (in red in the paper electronic version) and on the $\psi$-metallicity plane (in blue in the paper electronic version) respectively. The CMD of the mock population (mCMD)is shown on the right panel. Grey levels show the density of stars. A factor of two in density exists between each two successive gray levels.
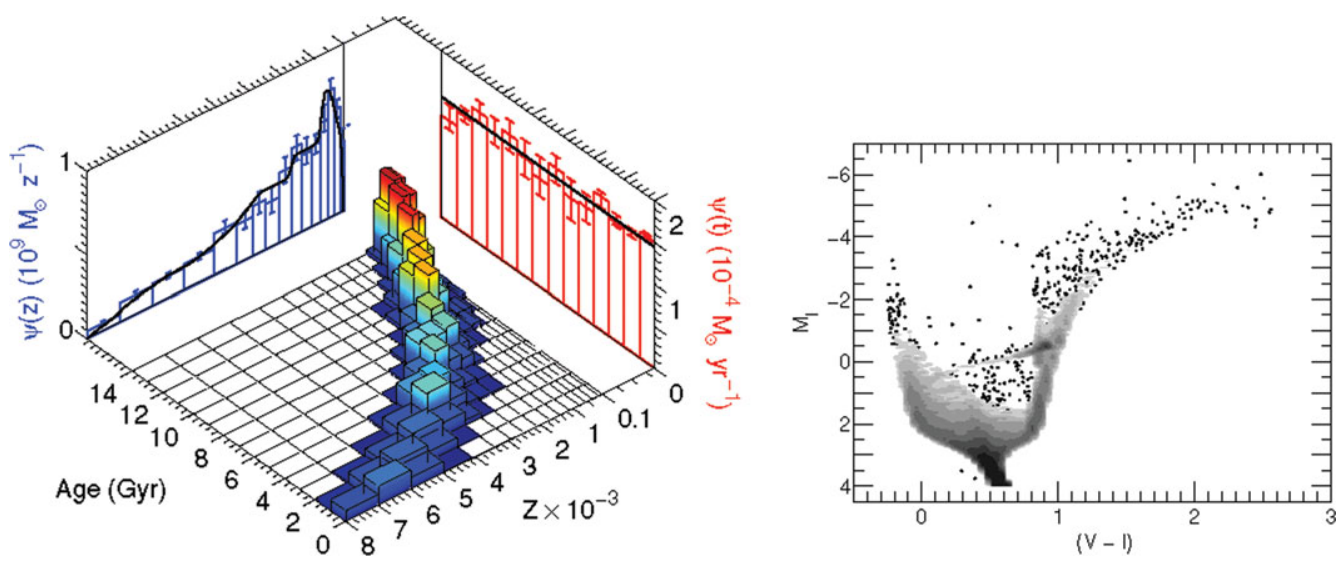

Figure 2. Solution of $\psi(t, z)$ obtained for the mock population (left panel). The caption is the same as in Figure 1. The CMD corresponding to the solution is shown on the right panel. To be compared with the mCMD shown in Figure 2.

over 24 orbits and also added to the LCID sample. The final objective is to analyze how star formation has proceeded in isolated dwarfs, free of strong tidal effects.

The galaxies were observed in F475W and F814W ACS filters (except Phoenix which was observed in F555W and F814W WFPC2 filters). The photometry list was obtained using DOLPHOT (Dolphin (2000)) (the photometry of Phoenix was obtained using DAOPHOT/ALLFRAME (Stetson, 1994)). The process described in this paper was used to obtain the preliminary SFHs of all galaxies, which are shown in Figure 3. Preliminary results show that all galaxies except LeoA have old (> $10 \mathrm{Gyr}$ ) star formation. The first star formation even seems to be stronger in Tucana and Cetus than the other galaxies. LeoA shows a delay in the first star-formation event. However, in the case of LeoA, our 


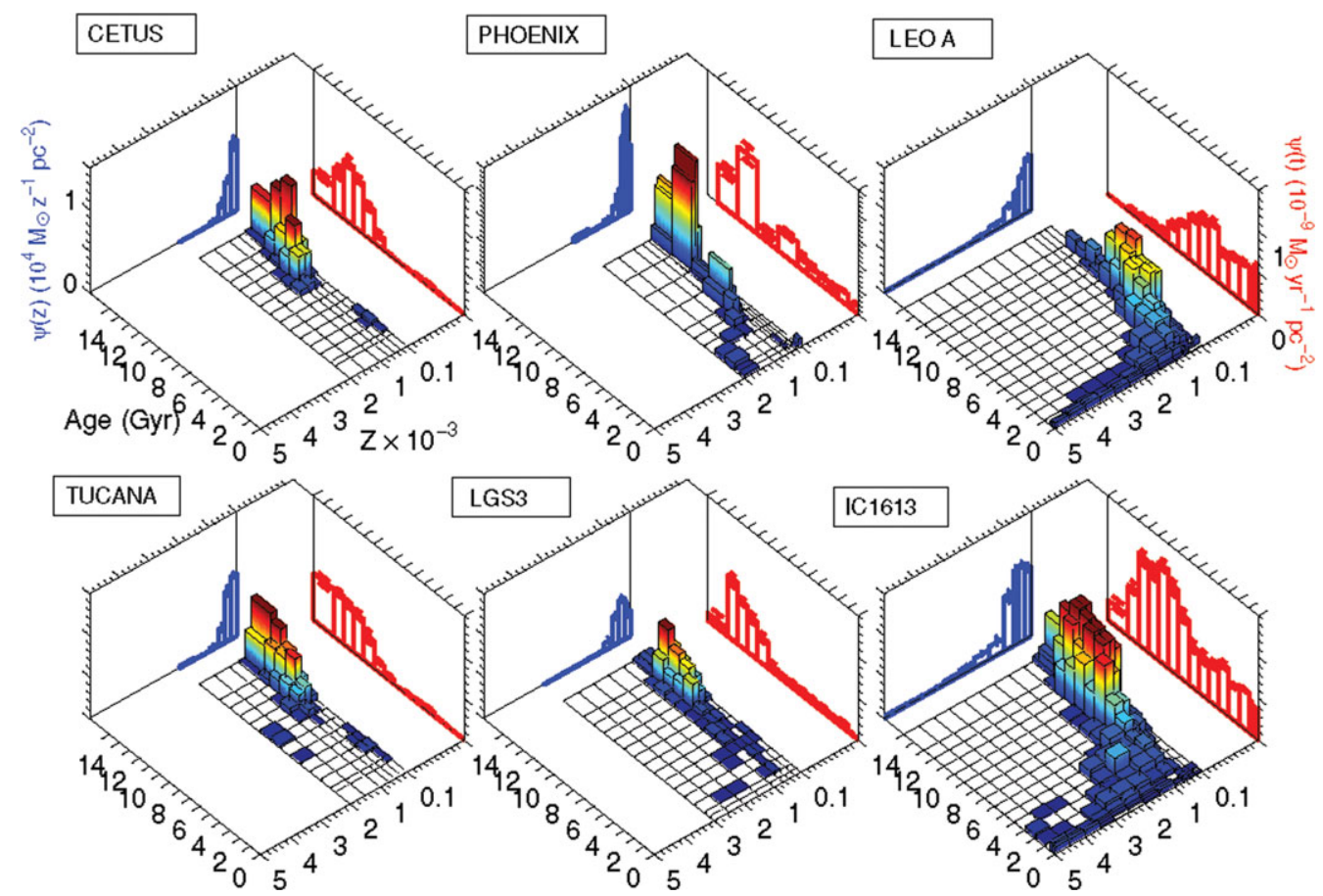

Figure 3. SFHs obtained for the six galaxies of the LCID project. The caption is the same as in Figure 1.

results show that the SFR for stars older than 10 Gyr depends on the distance and reddening assumed.

\section{Conclusions}

We have presented several algorithms which have been shown to be a useful tools to obtain the SFH from the CMD of resolved stellar systems. The procedure has been run through a consistency test showing the robustness of the method against the uncertainties introduced by realistic observational effects.

The method has been applied to obtain the SFHs of a set of six Local Group dwarf galaxies from the LCID project.

\section{References}

Aparicio, A. \& Gallart, C. 2004, AJ, 128, 1465

Aparicio, A., Gallart, C., \& Bertelli, G. 1997, AJ, 114, 669

Aparicio, A. \& Hidalgo, S. L. 2008, AJ, submitted

Castelli, F. \& Kurucz, R. L. 2003, New Grids of ATLAS9 Model Atmospheres IAU Symposium

Charbonneau, P. 1995, ApJS, 101, 309

Dolphin, A. E 2000, PASP, 112, 1383

Kroupa, P., Tout, C. A., \& Gilmore, G. 1993, MNRAS, 262, 545

Mighell, K. J. 1999, ApJ, 518, 380

Pietrinferni, A., Cassisi, S., Salaris, M., \& Castelli, F. 2004, ApJ, 612, 168

Stetson, P. B. 1994, PASP, 106, 250 


\section{Discussion}

M. SAlARIS: Given our current inability to predict HB Morphologies, what is the role played by the observed HB stars in your SFH determinations?

S. HidAlgo: We have not used HB stars at all. We use MS and sub-giant branch stars only.

M. Tosi: Could you comment on why Cole does find a (secondary) SF peak in Leo A at the earliest epochs while you have very little (if any) SF at those epochs?

S. Hidalgo: The star formation history derived by Cole et al. (2007, ApJ, 659, L17) uses a different stellar evolution library than this solution, which could introduce some differences between them. But, moreover, the secondary peak found by Cole is, within the error bars, compatible with the solution that I present here.

R. WySE: How robust is your result that star formation in Leo A did not start earlier than 10 Gyr ago? You mentioned that different stellar evolution models can give older ages.

S. Hidalgo: The solutions have been tested against several consistency tests. All the SFHs have been derived homogeneously. All other galaxies show evident SF older than 10 Gyr. Leo A presents a very low SF rate for that epoch, but there is something. We know that Leo A has an old population because there are RR-Lyrae stars in the galaxy. We tried to derive the SFH with two different stellar evolution libraries to look for the differences in the solutions. 


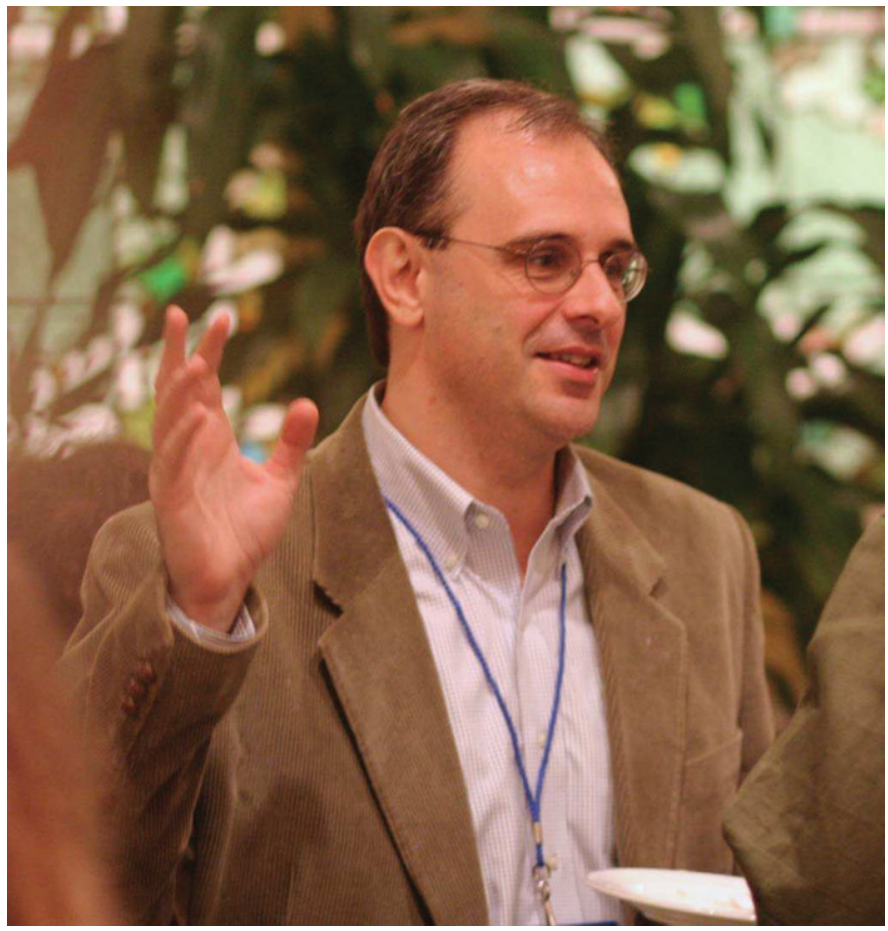

Enrico Vesperini

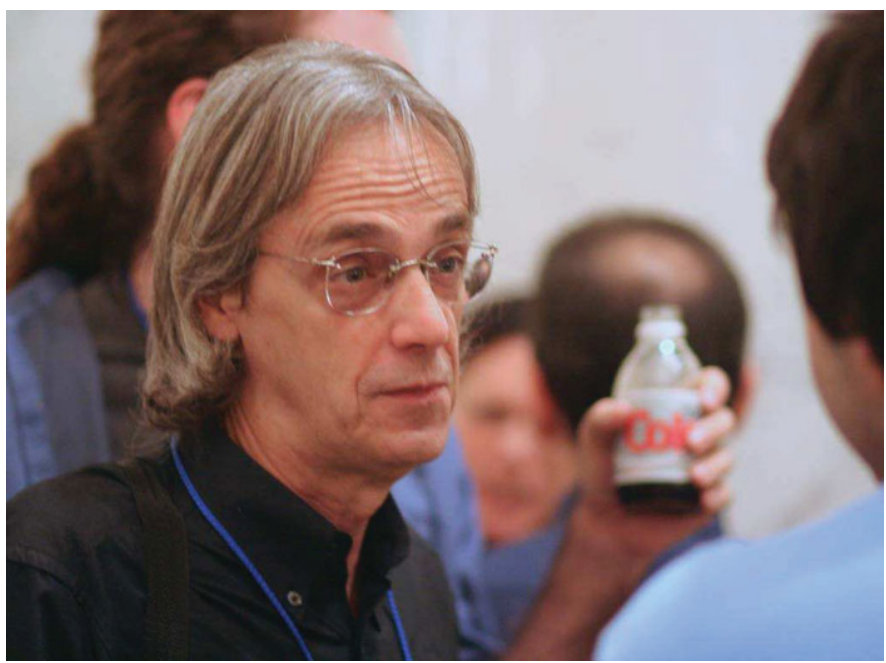

Walter Maciel 